\title{
Verzeichnis der Tabellen
}

1. Langhobelmaschinen. Seite

1. Werte iür schnellschnittstahl . . . . . . . . . . . . . 10

2. Durchschnittswerte iür Langhobelmaschinen . . . . . . . . 10

3. Darstellung einer Langhobelmaschine von $5 \mathrm{~m}$ Hobellänge . . 12

4. Darstellung "einer l.anghobelmaschine von $2.5 \mathrm{~m}$ Hobellänge . . 12

5. Aufspannzeiten an Hobelmaschinen . . . . . . . . . . . 15

6. Durchschnittswerte iür Schnittgeschwindigkeit und Vorschub 20

7. Ausgerechnete Werte fïr Schnittgeschwindigkeit und Hubzahl:

fïr Hobelmaschinen von $2 \mathrm{~m}$ gröBter Hobellänge . . . . 22

für Hobelmaschinen von $4 \mathrm{~m}$ größter Hobellänge . . . . 22

iür Hobelmaschinen von $8 \mathrm{~m}$ größter Hobellänge . . . . 22

8. - 8a. Ausgerechnete Laufzeiten . . . . . . . . . . . . . . . 23

\section{Shapingmaschinen.}

9a. Darstellung einer Shapingmaschine von $375 \mathrm{~mm}$ Hublänge . . . 26

9b. Werkstatt-Tabelle einer Shapingmaschine von $375 \mathrm{~mm}$ Hublänge 27

10a. Darstellung ciner Shapingmaschine von $300 \mathrm{~mm}$ Hublänge . . 28

10b. Werkstatt-Tabelle einer Shapingmaschine von $300 \mathrm{~mm}$ Hublänge 29

11. Auispannzeiten an Shapingmaschinen . . . . . . . . . 29

12. Durchschnittswerte für Schnittgeschwindigkeit und Vorschub

(Hub $=500 \mathrm{~mm})$. . . . . . . . . . . . . . . . 33

13. Ausgerechnete Doppelhübe iïr eine Hublänge bis $500 \mathrm{~mm}$. . . 34

14. Durchschnittswerte für Schnittgeschwindigkeit und Vorschub

(Hublänge bis $1000 \mathrm{~mm}$ ) . . . . . . . . . . . . . . 35

15. Ausgerechnete Doppelhübe für eine Hublänge bis $1000 \mathrm{~mm}$. . 36

16. Ausgerechnete Laufzeite॥ für eine Hublänge bis $500 \mathrm{~mm}$. . . 37

17. Ausserechnetc Laufzeiten fïr eine Hublänge bis $1000 \mathrm{~mm}$. . 38

\section{Sènkrechte StoBmaschinen.}

18a. Darstellung einer senkr. Stoßmaschine von $350 \mathrm{~mm}$ Hubhöhe. . 39

18b. Werkstatt-Tabelle einer senkr. Stoßmaschine von $350 \mathrm{~mm}$ Hubhöhe . . . . . . . . . . . . . . . . . . . 40

19. Auispannzeiten für kastenförmige Körper . . . . . . . . . 41

20. Aufspannzeiten für runde Körper . . . . . . . . . . . . 42 Graphische Vorschubitabelle fïr Kreisbewegung des Tisches . 45

IV. Drehbänke.

21. Umrechnung von Schnittgeschwindigkeiten . . . . . . . . 48

22. Umrechnung von Schnittgeschwindigkeiten . . . . . . . . 49

23. Minutliche Umdrehungszahlen . . . . . . . . . . . . . 50

23a. Minutliche Umdrehungszahlen . . . . . . . . . . . . . 51 
24. Schnelldrehstahl an Schnëlldrehbänken Seite

25. Schnelldrehstahl an gewöhnlichen Drehbänken . . . . . . . 53

26. Werte iür Nickel- und Chrom-Nickelstahl . . . . . . . . . 56

Graphische Tafel zur Bestimmung der minutl. Umdrehungszahl 58/59

27. Laufzeit für $10 \mathrm{~mm}$ Drehlänge bei $\mathrm{v}=10 \mathrm{~m} / \mathrm{Min}$. . . . . . 60 Graphische Laufzeittaiel . . . . . . . . . . . . . . 61

28. Drehbanktahelle; Aufstellung von Drehdurchmessern . . . . 64

29. Drehbanktabelle; Aufstellung von Drehdurchmessern . . . . 65

30. Das Schneiden von Flachgewinde . . . . . . . . . . . . 73

31. Das Schneiden von Spitzgewinde . . . . . . . . . . . . . . 74

32. Aufspannzeiten für Wellen . . . . . . . . . . . . . . 80

33. Aufspanrizeiten flir Buchsen . . . . . . . . . . . . . . . . . 81

34. Auispannzeiten für Ringe . . . . . . . . . . . . . . . . . . 82

35. Auispannzeiten iïr Scheiben . . . . . . . . . . . . . . . 83

\section{Revolverbänke.}

36. Zeiten für das Einrichten der Maschine . . . . . . . . . . . 85

37. Durchschnittswerte fïr Schnittgeschwindigkeit . . . . . . . 75

38. Durchschnittswerte fïr den Vorschub . . . . . . . . . . . 86

39. Durchschnittszeiten für das Umschalten der IVerkzeuge . . . 85

\section{Wagerecht-Bohrwerke.}

40. Durchschnittswerte für Schnittgeschwindigkeit und Vorschub 92

41. Aufspannzeiten fïr kastenförmige Körper . . . . . . . . . . 93

42. Aufspannzeiten fïr runde Körper (Buchsell) . . . . . . . . . . 94

\section{Rundschleifmaschinen.}

43. Durchschnittswerte für das Schleiien auf Maß . . . . . . . . 101

44. Durchschnittswerte fïr das Schleifen von Lagerstellen . . . . 103

\section{Fräsmaschinen.}

45. Durchschnitts-Schnittgeschwindigkeiten für Walzen- und Stirniräser . . . . . . . . . . . . . . . . . . . 104

46. Minutliche Vorschübe für mittlere Fräsmasch. (Schnellstahl) 105

47. Minutliche Vorschübe für größere Fräsmasch. (Schnellstahl) . 106

48. Minutl. Vorschübe für mittlere Fräsmasch. (Werkzeug-Gußstah!) 108

49. Minutl. Vorschübe für größere Fräsmasch. (Werkzeug-Gußstahl) 109

50. Zusatzwerte für Stirnfräser und Messerköpfe . . . . . . . 110

51. Aufspannzeiten für kastenförmige Körper . . . . . . . . . 111

52. Minutliche Vorschtabe für Nutenfräser (Schnellstahl) . . . . . 114

53 Minutliche Vorschübe für Nutenfräser (Gußstahl) . . . . . 115

54. Minutliche Vorschübe für Kreissägen . . . . . . . . . . 116

55. Minutliche Vorschübe für Winkelfräser . . . . . . . . . 117

56. Minutliche Vorschübe für Rundiräsmaschinen . . . . . . . 118 Das Fräsen von Stirnrädern.

57. Minutl. Umdrehungszahlen für Stirnradfräser (Teil- u. Wälzverf.) 120 


$$
\begin{aligned}
& \text { Tabelien für das Teilverfahren: Seite } \\
& \text { Material des Werkstückes Material des Frăsers }
\end{aligned}
$$

60 Nickelstahl, S.-M.-Stahl und Stahlguß

62. S.-M.-Stahl und Stahlguß von $50-60 \mathrm{~kg}$

64. Chrom-Nickelstahl von $80 \mathrm{~kg}$ Pestigkeit Schnellschnittstahl. 131

65. Chrom-Nickelstahl von $70 \mathrm{~kg}$ Festigkeit Schnellschnittstahl. 132

66. Nickelstahl, S.-M.-Stahl und Stahlguß von $50-60 \mathrm{~kg}$ Festigkeit .. . . Schnellschnittstahl . 133

67. weicher Stahl, Gußeisen und Bronze Schnellschnittstahl. 134

68. S.-M.-Stahl und Stahlguß von $50-60 \mathrm{~kg}$

Festigkeit . . . . . . . . . . Werkzeug-GuBstahl 135

69. weicher Stahl, GuBeisen und Bronze Werkzeug-GuBstahl 136

70. Zahn- und Frästiefen an Stirn- und Schneckenrädern . . . . 137

71. Fräslängen an Schnecken . . . . . . . . . . . . . . . 138

72. Minutliche Vorschübe beim Fräsen von Schnecken . . . . . 138

73. Durchschnittswerte f. d. Fräsen v. Schneckenrădern (tangential) 142

74. Durchschnittswerte f. đ. Fräsen v. Schneckenrädern (radial) . 144

\section{Bohrmaschinen.}

75. Lauf- und Gesamtzeit beim Bohren . . . . . . . . . . . 146

76. Durchschnittswerte für Schnittgeschwindigkeit und Vorschub 147

77. Umdrehungszahlen und Vorschübe für Schnellschnittstahl . . . 148

78. Umdrehungszahlen und Vorschübe für Schnellschnittstahl . . . 149

79. Umdrehungszahlen und Vorschübe für Werkzeug-OuBstahl . . 150

80. Aufspannzeiten für kastenförmige Körper . . . . . . . . . 151

81 Uebersichtstabelle für Schnittgeschwindigkeiten . . . . . 156 
Umdrehungen $\mathrm{i} d$

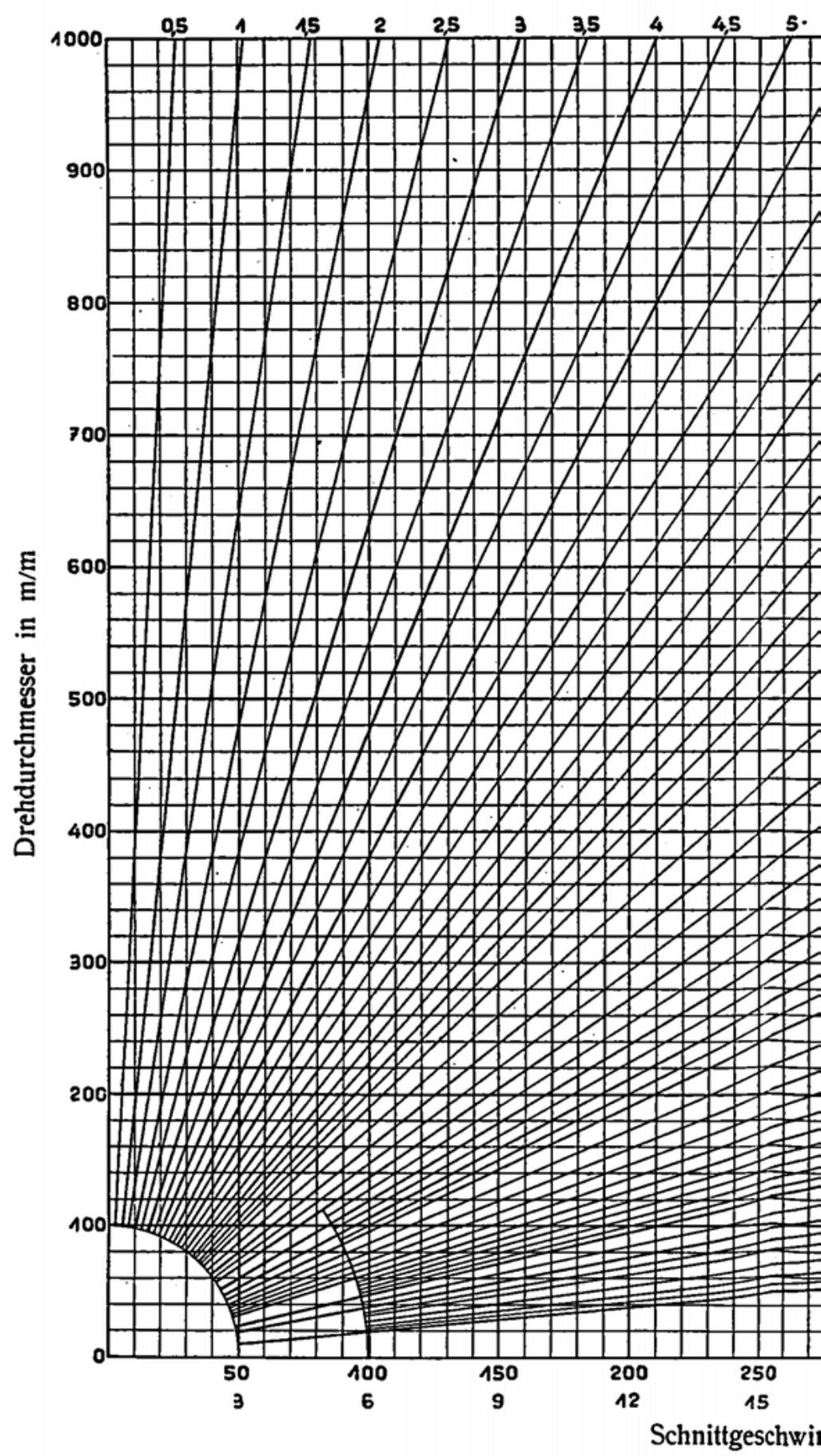

Fig. 23 
